\title{
PROCESOS DE ORIENTACIÓN EN ENTORNOS VIRTUALES DE APRENDIZAJE
}

\author{
COUNSELLING IN VIRTUAL LEARNING ENVIRONMENTS
}

\author{
Antonio Pantoja Vallejo* \\ Universidad de Jaén \\ Marlene Zwierewicz** \\ Universidade UNIBAVE - Orleans (Brasil)
}

\begin{abstract}
RESUMEN
La historia de la humanidad se desarrolla de forma paralela a la expansión de las nuevas tecnologías. Su uso en la educación y la orientación deberá permitir condiciones para la atención personalizada del alumnado que accede a los sistemas digitales desarrollados al efecto, como es el caso de los Entornos Virtuales de Aprendizaje (EVA).

En el presente artículo se analizan estas cuestiones desde una perspectiva orientadora, se valora la interactividad que favorece los EVA, se estudian las características que presentan el orientador y el tutor virtual y se realizan aportaciones desde un punto de vista prospectivo.
\end{abstract}

Palabras clave: Entornos virtuales de aprendizaje, orientación, nuevas tecnologías, tutor, orientador.

\begin{abstract}
The history of human civilization runs parallel to the expansion of the new technologies. Their use in education and counselling must facilitate the necessary conditions for personalized attention to students gaining access to digital systems developed for this purpose, such as Virtual Learning Environments (EVE, in Spanish).

This paper discusses these issues from a counselling perspective, it assesses interactivity fostered by EVE, reviews counsellors characteristics and virtual tutors, and contributes to the field from a prospective point of view.

Key words: Virtual learning environments, counselling, new technologies, tutor, counsellor.

\footnotetext{
* Facultad de Humanidades y CC. de la Educación. Departamento de Pedagogía, Área MIDE • Despacho 242. Campus las Lagunillas, s/n Correo-E: mailto:apantoja@ujaen.es

** Universidade UNIBAVE - Orleans (Brasil). Correo-E: marlenezwie@febave.org
} 


\section{Introducción}

En 1995 Rodríguez Diéguez afirmaba que las nuevas tecnologías aplicadas a la educación tenían como finalidad la capacitación del futuro profesor como usuario de recursos multimedia (p. 33). Eran estos, sin duda, los comienzos de la introducción de las Tecnologías de la Información y la Comunicación (TIC) en el entorno educativo y en la sociedad en general. Apenas se escuchaba por entonces el término Sociedad de la Información (SI) (Pantoja, 2004), que iba a definir el nuevo escenario que se empezaba a formar.

Todos estos años no han hecho sino poner de manifiesto que el camino ya trazado por Rodríguez Diéguez sigue su curso, pero mucho más lento de lo que cabría esperar. Así todavía hablamos de "nuevas" — como él consideraba a las tecnologías — más de una década después, tal y como se ha puesto de manifiesto en una investigación llevada a cabo por la Fundación BBVA en octubre de 2005 en la que se constata que sólo uno de cada tres hogares españoles es usuario de Internet (http://www.fbbva.es).

El dato anterior sobre la tecnología de moda no hace sino ahondar en el panorama de la llegada de las TIC a todos los rincones de la sociedad, favoreciendo la aparición de formas inéditas en las relaciones humanas y en la convivencia de las personas y de los grupos. Al mismo tiempo, se producen posibilidades de intervención e intercambio de experiencias culturales entre usuarios en entornos virtuales hasta ahora desconocidos.

En educación y en orientación, nos encontramos ante la posibilidad de revolucionar estrategias y superar prácticas centradas en la relación unilateral emisor-mensaje-receptor, caracterizadas por el aprendizaje memorístico de los contenidos, por la centralización del conocimiento en el profesor y por la ausencia de interactividad.

La utilización de las TIC posibilita formas diferentes de enseñanza y aprendizaje, protagonizando un proceso de inclusión y de respeto al universo cognitivo y emocional particular del alumno. Mientras, la realidad nos lleva a una dificultad evidente para hacer efectivas tales estrategias, principalmente en países con índices elevados de exclusión digital (otro de los motivos por lo que seguimos hablando de "nuevas" al referirnos a las tecnologías).

Para superar este panorama y llegar a una propuesta inclusiva, además de posibilitar un acceso generalizado a los recursos y a la red, también es preciso conseguir oportunidades de interactividad en los diferentes medios. Cualquier propuesta de este tipo debe ser permanentemente evaluada por los efectos producidos en el aprendizaje de los alumnos. Diferentes autores indican los aspectos que deben ser considerados en el diseño y aplicación de herramientas virtuales de aprendizaje, permitiendo una visión más amplia sobre la posibilidad de interactividad en los nuevos recursos tecnológicos, los criterios para evaluar las herramientas y el acompañamiento permanente de los alumnos.

Así, Silva (2003) selecciona elementos que considera imprescindibles para que las nuevas herramientas favorezcan la interactividad: la participación colaborativa; el proceso en el cual participar no es simplemente contestar sí o no o elegir una opción cerrada; la bidireccionalidad en la que comunicación y producción van conjuntas; por fin, las conexiones en tareas abiertas en las que la comunicación señala múltiples redes de conexiones y libertades de cambios, asociaciones y significaciones.

De igual forma, las herramientas de enseñanza y aprendizaje a distancia han de ser evaluadas para conocer su verdadera utilidad. Según Cabero (2001), se podrá realizar a partir de algunos criterios: contenidos, aspectos técnicos, organización interna de la información, 
material de acompañamiento, costo económico, ergonomía del medio, aspectos físicos y público al cual se destina.

Al margen de la evaluación específica de la parte formativa de la aplicación, orientada a la validación de su uso, el diseño debe contemplar la evaluación permanente de los alumnos en tres niveles: diagnóstica, formativa y sumativa. Diagnóstica porque permite tener acceso a los conocimientos previos de los alumnos, detectar posibles limitaciones, estilos y ritmos de aprendizaje. Formativa, por facilitar el autoconocimiento y la intervención. Y, finalmente sumativa por permitir la interlocución entre los actores de los procesos formativos.

Entre propuestas inclusivas y orientadoras, se apuntan en este artículo algunos aspectos técnicos y pedagógicos que precisan ser contemplados cuando se proyectan programas de enseñanza en entornos virtuales.

\section{Entornos virtuales de aprendizaje}

La historia de la humanidad se desarrolla de forma paralela al avance de la tecnología. Al fabricar los instrumentos que posibilitan sus acciones, el hombre favorece la realización de sus actividades y transforma la realidad. En ese sentido, la técnica es producida por el hombre al mismo tiempo que ésta permite cambios del comportamiento humano que ayudan a su uso y a las relaciones humanas establecidas entre los hombres cuando la utilizan.

Para Lévy (2001) es imposible separar al ser humano de su ambiente material, pues una técnica es producida dentro de una cultura y la sociedad se encuentra condicionada por sus técnicas. Como tampoco se puede separar el mundo material del mundo de las ideas, por medio de las cuales los objetos técnicos son concebidos y utilizados por los humanos que los inventan y utilizan.

Esta fuerte relación se tornó más perceptible a partir de la década de 80 cuando Internet permitió la conexión de un gran número de ordenadores, pero de manera especial fueron los años 90 los que la extendieron a todo el planeta, constituyéndose en un nuevo entorno para el intercambio de informaciones.

A partir de la nueva configuración de los espacios comunicativos, Oliveira (2003) argumenta que se vive un periodo de transición muy importante en la trayectoria de la humanidad en el que todo cambia en una compleja red de relaciones. Un periodo en el que los cambios provienen de la rapidez de los aconteceres, de la simultaneidad de los hechos y más importante aún, de la coexistencia de los hechos y la comunicación.

El momento de transición es marcado principalmente por la creciente democratización de la informática y la difusión de la cibercultura, lo que facilita la aparición de formas distintas de relaciones entre los sujetos. Éstas ocurren en tiempo real o diferido y superan dificultades impuestas por las fuerzas naturales como el espacio geográfico y el tiempo.

Castillo (2001) afirma que es cada vez más palpable que las TIC están cambiando el mundo, desde las relaciones de producción hasta los modos de ocio, transformando la organización social y las representaciones y conductas individuales.

En medio de las acciones humanas organizadas a lo largo de la historia de la humanidad, la educación formal se encuentra desarrollada en entornos presenciales de aprendizaje, sin embargo, las nuevas posibilidades que nos ofrecen las TIC favorecen la construcción de 
espacios educativos sin las limitaciones convencionales de espacio y tiempo de la educación presencial.

Tales espacios educativos, encuadrados dentro de lo que se conoce como Entornos Virtuales de Aprendizaje (EVA), se estructuran sin la necesaria presencia física de alumnos, profesores y/o orientadores y permiten la flexibilidad de horarios y desarrollan nuevas habilidades técnicas y cognitivas para recibir, criticar y manipular las informaciones de forma compartida.

Santos (2003) afirma que el ambiente virtual es un espacio fecundo de significación donde los seres humanos y objetos técnicos interactúan, potenciando la construcción del conocimiento. Por su parte, Salinas (2004: 471) matiza el término EVA añadiéndole también enseñanza y lo concreta como aquel "escenario físico donde un alumno o comunidad de alumnos desarrollan su trabajo, incluyendo todas las herramientas, documentos y otros artefactos que pueden ser encontrados en dichos escenarios".

Por nuestra parte definimos los EVA de la siguiente forma, completando otra realizada con anterioridad (Zwierewicz y Pantoja, 2004: 2):

"Espacios de aprendizaje dominados por las TIC que permiten una simulación en tiempo real de las condiciones que se dan en un aula presencial y que ofrecen condiciones técnicas para el desarrollo de estrategias interactivas y la consecuente construcción colaborativa del conocimiento, aunque en este caso docente y discente se pueden encontrar a miles de kilómetros de distancia".

En los EVA los individuos conectados pueden apropiarse de nuevos conocimientos generados en procesos de análisis y reflexión colaborativa y superar la posición de simples espectadores del mensaje.

La utilización de los referidos entornos posibilita la aparición de estrategias que permiten establecer un nuevo paradigma de enseñanza, superando así prácticas excluyentes caracterizadas por el aprendizaje memorístico de los contenidos y por la ausencia de interactividad. Se promueven propuestas inclusivas, en las que se priorizan las prácticas interactivas para que los sujetos conectados puedan intervenir y construir el conocimiento de forma cooperativa.

Además, las mismas formas de trabajo que posibilitan los recursos informáticos permiten la creación de ambientes en los cuales el proceso de enseñanza y aprendizaje favorece el acceso a un número de informaciones históricamente desconocido y la interacción de conocimientos de las más variadas culturas.

Para que esto sea una realidad es necesario que el sistema educativo sea considerado como un todo que permita no solo el uso de nuevas herramientas, sino también la producción de aprendizaje y la generación de esquemas de organización que deben ser analizados y comprendidos por sí mismos para valorar sus efectos (López, 2004). En este punto, conviene recordar que en él existen problemas que han surgido y que aún no se resolvieron por completo, entre los cuales destaca la posibilidad de acceso a las TIC que se sitúa en parámetros de exclusión digital; el estímulo a la cibercultura; y la necesidad de creación de estrategias interactivas para el intercambio de los sujetos conectados en los EVA, teniendo en cuenta los diferentes medios disponibles y las características de los participantes, así como la formación previa de los alumnos para el desarrollo de habilidades y competencias para trabajar con las TIC. 


\section{Orientar en la Sociedad de la Información (SI)}

La SI es "una forma de evolución social basada en el uso habitual de las Tecnologías de la Información y la Comunicación (TIC) — habrán dejado de ser nuevas para la mayoría de la colectividad mundial - por todos los ciudadanos a nivel individual y colectivo, público y privado, para obtener, tratar y compartir información de forma instantánea desde cualquier lugar, tiempo y forma definidos previamente por sus usuarios" (Pantoja, 2004: 127).

La orientación en el ámbito educativo tiene ante sí un desafío de enorme calado dentro de la SI, que corre paralelo a otras instancias constituyentes de la misma. Los desarrollos tecnológicos permitirán el diseño y el uso de potentes herramientas en el proceso de aprendizaje y mantener un contacto más cercano al alumnado y a las familias, entre otros. Se puede afirmar que los mismos retos de la educación y de los subsistemas sociales (Negroponte, 1995; Terceiro, 1996; Fernández, 1998; Levy, 1999) son igualmente válidos para la orientación. Aplicaciones como las representaciones virtuales, la capacidad interactiva, la simulación en tiempo real, su enfoque global o su proyección hacia las relaciones interpersonales y la participación estarán en manos de los orientadores y facilitarán enormemente su trabajo.

Principios como la consecución de un servicio universal basado en la no discriminación y la igualdad en acceso (Delors en su informe de 1996 se refirió a este tema), la producción de contenidos culturales que alimenten las redes de ocio y enseñanza, la ampliación de contenidos que den respuestas a las distintas áreas de la orientación o la mejora de la calidad de las distintas ofertas orientadoras que se produzcan, deberán ser asumidos por la SI.

En el estado español, los nuevos centros (TIC) que se están configurando en todo el territorio nacional cuentan con abundantes recursos tecnológicos y suponen una buena respuesta a las demandas de innovación que provienen de la SI; no obstante, todavía están lejos de generalizarse. En ellos, el orientador tiene la responsabilidad de adecuar sus tareas y funciones a las posibilidades que la red le ofrece de mantener una comunicación más directa y constante con los estudiantes.

Sin lugar a dudas serán las redes telemáticas las llamadas a comandar el proceso orientador bajo el dominio y supervisión del profesional especializado. Este supuesto nos lleva a hablar de e-orientación como "el conjunto de iniciativas que persiguen explotar las posibilidades de la orientación dentro del espacio electrónico promovido por las redes telemáticas, con un predominio del procesamiento digital de la información y del conocimiento" (Pantoja, 2004: 148-149). Para que llegue a ser una realidad habrán de ser necesarios una serie de cambios de tipo material (mejora de infraestructuras y dotaciones materiales), formativo (dominio de las TIC por parte de orientadores y tutores), metodológico (creación de estructuras dentro de los centros que precisen del uso de las TIC) y conceptual (idea de la educación y de la orientación futuras consensuada por los expertos en el tema e implementada en los propios contextos de la orientación). Es decir, para que la orientación basada en las TIC sea una firme apuesta de futuro hará falta que exista una demanda concreta de sus usuarios y precisará de profesionales bien formados que puedan hacerla realidad.

Si las previsiones europeas se mantienen, se abrirá en los centros educativos un espacio virtual de información y comunicación en el cual la e-orientación habrá de diseñar nuevos procesos de orientación y tutoría adaptados a la realidad palpable de los centros (Campoy y Pantoja, 2003), de manera que sea posible trabajar e investigar en los mismos (coordinación entre tutores y orientador) y fuera de ellos, en todas las cuestiones relacionadas con aspectos personales, sociales, familiares, académicos y profesionales. 


\section{E-escuela, orientador y tutor virtual}

La e-escuela es el espacio en el que desarrollarán su labor los orientadores y tutores digitales, se trata de un nuevo entorno de y para el cambio, heredero de los procesos tradicionales de educación y orientación y en la que se promueven diversos tipos de contactos entre educando y educador, entre orientado y orientador, entre padres y tutor. Se puede definir de la siguiente forma (Pantoja, 2004: 177):

"...entorno de aprendizaje en el que prevalecen un conjunto de acciones y de relaciones generadas por el uso eficaz, continuado e integrado en la práctica docente y orientadora de las redes digitales y las herramientas asociadas a las mismas".

Este nuevo concepto de educar tiene ante sí una serie de cambios dirigidos al uso y gestión de las opciones comunicativas de la SI. Éstas, afectan por igual a todos los implicados en el aprendizaje, pero de manera especial a los profesionales y al alumnado.

Por tanto, la e-escuela se constituye en un tipo de EVA que ofrece distintas connotaciones y características dependiendo de las variables que rodeen cada centro concreto y de las posibilidades técnicas disponibles dentro y fuera del mismo (familias). Este segundo aspecto es vital, pues de él depende la firmeza del puente que las redes telemáticas tiendan entre centro y hogares.

Pensamos que las características que deberán rodear al orientador y al tutor virtual de la e-escuela serán coincidentes en parte con las existentes en la e-universidad, e-empresa, eacademia, etc., aunque cada modalidad de Educación a Distancia $(\mathrm{EaD})$ creará sus propios EVA y en ellos se definirán funciones y tareas específicas de las personas responsables de orientar a distancia a cada grupo de alumnos.

El hecho de que las redes telemáticas sean ya una realidad en muchos centros educativos está permitiendo un tipo de orientación diferente, un apoyo de la acción orientadora directa en la virtual (Pantoja, 2006). Esta nueva alternativa orientadora provoca cambios en los roles y funciones de los orientadores (cuadro 1).

CUADRO 1. Diferentes funciones desarrollados por el orientador virtual.

\section{Orientador virtual}

- Diseña, desarrolla y evalúa programas de intervención basados en las TIC (Modelo tecnológico).

- Dinamiza y gestiona a través de la intranet del centro las distintas relaciones entre los miembros de la comunidad educativa relacionadas con la orientación.

- Busca información, la interpreta y la pone en la red a disposición de los orientados.

- Resuelve problemas de forma indirecta a través de los materiales que ofrece en su Web.

- Investiga en la red.

- Coordina a los tutores en su relación virtual con el alumnado.

- Propone contenidos tecnológicos de interés y los ofrece organizados de acuerdo a las distintas áreas de la orientación.

- Busca nuevas relaciones entre clientes.

- Organiza actividades en red. 
Con respecto al tutor virtual, éste será en los centros educativos también tutor presencial, por lo que deberá mantener sus competencias en el uso de técnicas como la dinámica de grupos, la observación o la entrevista. Esta última puede adoptar un formato presencial y virtual a través de la videoconferencia, y el tutor habrá de saber compaginar ambas modalidades. En la entrevista con padres de alumnos, el tutor ha de conseguir que el contenido y la profundidad de las preguntas y las respuestas obtenga resultados similares a si estuvieran ante él. Ahora bien, siempre que sea posible es preferible el trato directo.

En el cuadro 2 se recogen las principales características del tutor virtual.

CUADRO 2. Características del tutor virtual.

\section{Tutor virtual}

- Conoce las teorías y principios que sustentan el aprendizaje a distancia.

- Domina las herramientas telemáticas que permiten la comunicación con sus tutelados: e-mail, videoconferencia, foro, páginas Web, etc.

- Posee competencias para gestionar las herramientas anteriores (actualizar, mantener, mejorar, etc.). Por ejemplo, es capaz de usar el Messenger y administrar una conversación entre él y varios de sus tutelados, permitiendo verse las caras al mismo tiempo.

- Es un experto en el uso de la comunicación telemática.

- Tiene capacidades divergentes para saber qué herramientas telemáticas son las más adecuadas a cada tipo de aprendizaje y a cada persona.

- Ha adaptado las técnicas tutoriales a las características del EVA específico sobre el que se sustente el aprendizaje. Por ejemplo, no es igual realizar una entrevista tutorial en un curso a distancia que a unos padres que no pueden asistir de forma presencial a la tutoría de su hijo.

- Cuenta con un amplio repertorio de recursos en red, algunos de ellos específicos como las simulaciones.

- Diseña y pone en práctica un Plan de Acción Tutorial que cuenta con apartados desarrollados a distancia.

- Conoce y utiliza instrumentos de evaluación propios de la EaD, que le permiten tanto el seguimiento individualizado como la gestión del grupo.

Orientador y tutor virtual mantienen parecidas competencias en el uso de determinadas herramientas de comunicación. A modo de ejemplo, pueden servir las delimitadas por Cogoi (2002: 6) en relación con los diferentes ámbitos en los que la videoconferencia puede resultar eficaz en la orientación: formación, asesoramiento/orientación individual y de grupo, y entrevistas de selección. Otros estudios (Sánchez Arroyo, 2001) señalan una cierta desinhibición de la persona que no está de forma presencial, por lo que pueden aflorar actitudes y reacciones no habituales. ¿Podrá gestionarlas el orientador y/o el tutor? Ciertamente es complejo adelantar que sea así, toda vez que los tutores actuales están formados para atender a personas de forma presencial y todavía no cuentan con tradición en este nuevo rol. Lo realmente importante, es que la interacción no se pierda en las relaciones a distancia.

\section{A modo de conclusión}

Aún sabiendo que los EVA precisan de desarrollos específicos adaptados a cada campo de conocimiento, y de investigaciones que pongan de manifiesto sus ventajas con respecto a 
los sistemas presenciales tradicionales, no es menos cierto que, en nuestro caso, se necesitan definir las aportaciones concretas que pueden hacer a la orientación. Algunas de ellas, a modo de ejemplo, son:

- Forma en la que el alumno aprende en los EVA: presentar los objetivos del aprendizaje y un esquema general de los contenidos, incluir mapas de navegación, ofrecer una guía al alumno que le informe sobre aspectos metodológicos, presentar los contenidos, ofrecer recursos, presentar actividades y evaluar (Pérez y Salinas, 2004).

- Promoción de la investigación en la red como eje vertebrador de los procesos de orientación en el alumnado: creación y la realización de WebQuest temáticas ${ }^{1}$.

Para finalizar, señalamos que los EVA cuentan todavía, como hemos visto, con una escasa experiencia en el mundo educativo y, menos aún, en el de la orientación. Los diferentes beneficios que puedan aportar, el uso que los orientadores y tutores hagan de los mismos, junto con la calidad de los desarrollos técnicos que vayan apareciendo, deberán ir acompañados de la reflexión permanente de todas las personas implicadas, sean orientadores, técnicos, educadores o alumnos. Por el contrario, podremos utilizarlos como simples instrumentos, no explorando las nuevas estructuras que los mismas ofrecen para la convivencia de pluralidades a través de la interactividad.

Los próximos años servirán para poner de manifiesto estudios en los que aparezcan las necesidades reales para orientar y tutorizar en los EVA. La pregunta es ¿están el orientador y el tutor preparados para ello? Por ahora no hay respuesta para esta interrogante, aunque si nos atenemos a la configuración de los planes de estudio de las distintas titulaciones, las perspectivas no son nada halagüeñas.

\section{Referencias bibliográficas}

Cabero, J. (2001). "Evaluar para mejorar: medios y materiales de enseñanza". En J. M. Sancho (coord.), Para una tecnología educativa (pp. 241-276). Barcelona: Horsori.

Campoy, T. J. y Pantoja, A. (2003). "Propuestas de e-orientación para una educación intercultural". Comunicar, 20, 37-43.

Castillo, J. L. (2001). "Posibilidades y límites político-educativos de las nuevas tecnologías de la información y la comunicación". Revista Española de Pedagogía, 218, 85-104.

Cogoi, C. (2002). "Videoconferenza e orientamento". Revista Española de Orientación y Psicopedagogía, 13 (1), 5-15.

Delors, J. y otros (1996). La educación encierra un tesoro. Madrid: Santillana.

Fernández, L. A. (1998). En.red.ando. Barcelona: Zeta Ediciones.

Levy, P. (1999). ¿Qué es lo virtual? Barcelona: Paidós.

Lévy, P. (2001). Cibercultura. São Paulo: Editora 34.

López, J. M. T. (2004). "La Educación electrónica: un reto de la sociedad digital en la escuela". Revista Española de Pedagogía, 227, 31-56.

Negroponte, N. (1995). El mundo digital. Barcelona: Ediciones B.

1. En la página Web http://www.antoniopantoja.com.es se puede encontrar un repertorio de WebQuest dedicadas a la orientación y la tutoría. 
Oliveira, E. G. (2003). Educação a distância na transição pradigmática. Campinas, SP: Papirus.

Pantoja, A. (2004). La intervención psicopedagógica en la Sociedad de la Información. Educar y orientar con nuevas tecnologías. Madrid: EOS.

Pantoja, A. (2006). "Acción tutorial y nuevas tecnologías". En M. Álvarez (coord.), La acción tutorial: su concepción y su práctica (pp. 219-261). Madrid: MEC.

Pérez, A. y Salinas, J. (2004). "El diseño, la producción y realización de materiales multimedia e hipermedia”. En J. Salinas, J. A. Aguaded y J. Cabero (coords.), Tecnologías para la educación. Diseño, producción y evaluación de medios para la formación docente (pp. 157-176). Madrid: Alianza Editorial.

Rodríguez Diéguez, J. L. y Sáenz, O. (dir.) (1995). Tecnología educativa. Nuevas tecnologías aplicadas a la educación. Alcoy: Marfil.

Salinas, J. (2004). Cambios metodológicos con las TIC. Estrategias didácticas y entornos virtuales de aprendizaje. Bordón, 56 (3 y 4), 469-481.

Santos, E. O. (2003). "Articulação de saberes na EAD online: Por uma rede interdisciplinar e interativa de conhecimentos em ambientes virtuais de aprendizagem". En M. Silva (coord.), Educação Online: teorias, práticas, legislação, formação corporativa (pp. 217-230). São Paulo: Edições Loyola.

Silva, M. (2003). "Criar e professorar um curso online: relato de experiência”. En M. Silva (coord.), Educação Online: teorias, práticas, legislação, formação corporativa. (pp. 51-73). São Paulo: Edições Loyola.

Terceiro, J. B. (1996).Socied@ddigit@l.Del homo sapiens al homo digitalis. Madrid: Alianza Editorial.

Zwierewicz, M. y Pantoja A. (2004). Diversidad e identidad en Ambientes Virtuales de Aprendizaje. En Actas del XIII Congreso Nacional y II Iberoamericano de Pedagogía "La educación en contextos multiculturales: diversidad e identidad". Edición en CD-Rom.

Fecha de recepción: 05-07-06

Fecha de revisión: 21-04-08

Fecha de aceptación: 21-05-08 